\title{
Active Separation Control at the Pylon-Wing Junction of a Real-Scale Model
}

\author{
Philipp Schloesser* \\ Airbus Defence and Space GmbH, 81663 Munich, Germany \\ Vitaly Soudakov ${ }^{\dagger}$ \\ Central Aerohydrodynamic Institute (TsAGI), 140180 Zhukovsky, Moscow Region, Russia \\ Matthias Bauer \\ Navasto GmbH, 12435 Berlin, Germany \\ Jochen Wild ${ }^{\S}$ \\ Deutsches Zentrum für Luft- und Raumfahrt e.V., 38108 Braunschweig, Germany
}

The effect of active flow control on local flow separation behind the installation location of an ultra-high-bypass-ratio nacelle is investigated in a real-scale experiment at the TsAGI T-101 wind tunnel. The investigated model represents a swept 2.5D-section of the pylon-wing junction in landing configuration. A flow control system employing periodic excitation is integrated into the unprotected leading edge inboard of the pylon. Tuft visualization as well as pressure and force measurements are used to investigate the vortex-dominated base flow and the effect of active flow control. It is shown that the flow control effect is governed by the normalized parameters of flow control, the momentum coefficient and the velocity ratio, and is largely independent of the free-stream Mach and Reynolds number within the investigated range. The influence of a variation of the momentum coefficient $c_{\mu}$ on the lift gain is investigated. At the highest Reynolds number of $R e=10.7 \cdot 10^{6}$ an application of active flow control with $c_{\mu}=1.08 \%$ fully eliminates local separation and increases total lift by approximately $2 \%$ of the respective baseline value across a range of 5 degrees in angle of attack.

\section{Nomenclature}

$$
\begin{aligned}
& C_{D}=\text { drag coefficient } \\
& C_{L}=\text { lift coefficient } \\
& c_{p}=\text { pressure coefficient } \\
& c_{\mu}=\text { momentum coefficient, }\left(\dot{m} \cdot u_{\text {jet }, \text { RMS }}\right) /\left(q_{\infty} \cdot S\right)
\end{aligned}
$$

\footnotetext{
*PhD Candidate, Department of Aeromechanics Systems, philipp.schloesser@airbus.com

†Senior Researcher, Department of Aerodynamics, vit_soudakov@tsagi.ru

\#Research Engineer, CEO, matthias.bauer@navasto.de

${ }^{\S}$ Team Lead High-Lift, Institute of Aerodynamics and Flow Technology, jochen.wild@dlr.de
} 


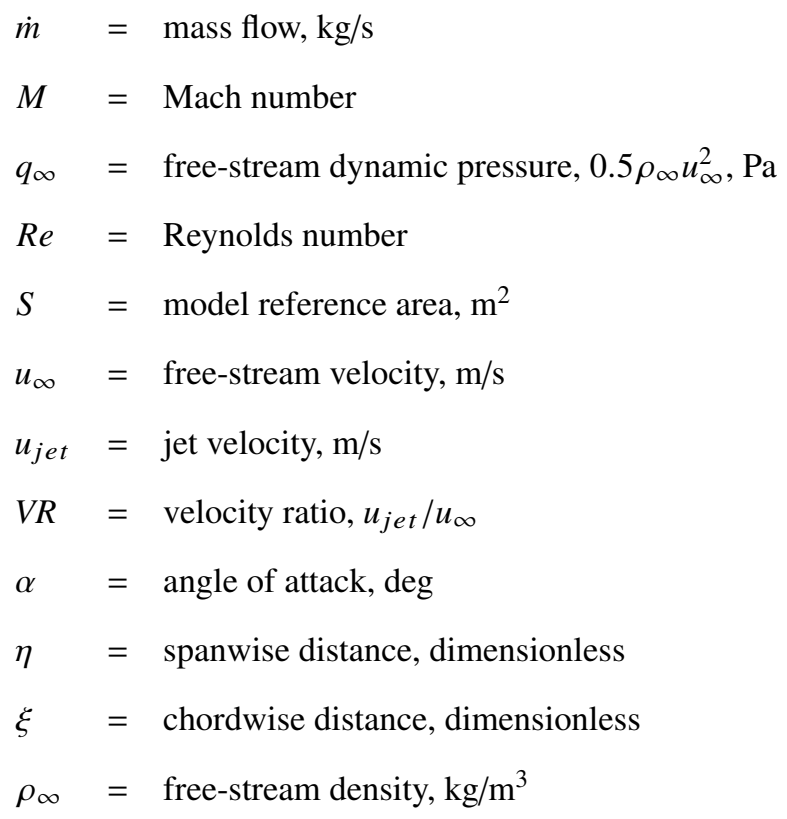

\section{Introduction}

Large modern civil transport aircraft are equipped with underwing-mounted turbofan engines that require a cutout of the leading edge high-lift system to prevent clashes with the engine nacelle. Highly efficient engines make use of large bypass ratios (BPR) to reduce fuel burn. Whereas currently employed engines reach BPRs of $10-12$, the trend in future development is heading towards even higher ratios of up to 15 -18 imposing installation challenges due to increased nacelle diameters. These so called ultra-high-bypass ratio (UHBR) engines need closer coupling to the wing to satisfy ground clearance requirements and to avoid a redesign of the landing gear. As a consequence, the slats have to be shorter than with smaller engines, which increases the area of the wing not protected by a leading edge high-lift system. The region downstream of this cutout is prone to separation due to an interaction of multiple vortices shed from the slat side-edge, pylon and nacelle [1-3]. On conventional aircraft, stall is commonly triggered by separation close to the wing root and in the region behind the slat cutout $[1,2]$. An increase of the slat cutout further weakens the flow in this area thereby limiting the maximum lift capability of the wing. Passive devices like strakes are a standard measure to counteract the separation $[4,5]$ but are limited in effectiveness with increased slat cutout and nacelle size.

An alternative to passive devices like strakes and movables is the technology of Active Flow Control (AFC). It has been subject of research on generic wings $[6,7]$, and has since moved to more complex configurations like outer wing sections $[8,9]$ or the vertical tail plane [10]. First flight test experiments with separation control on the vertical stabilizer of a large airliner show the capability of the technology on industrial scale [11]. Within the framework of the paper at hand, an industry relevant model has previously been tested with continuous blowing replacing a scaled $300 \mathrm{~mm}$ cutback of the inboard droop nose device [12]. The Reynolds number $R e=1.6 \cdot 10^{6}$ of the experiment is still 
within the range of scaling effects [13]. Nevertheless the application of AFC led to up to 5.6\% increase in maximum lift and delayed wing stall by up to 2.3 degrees [12]. Although the model and test conditions are not fully representative of the investigation at hand, the results show the compelling effect of local flow control at the pylon-wing junction. Unsteady excitation is commonly applied to increase the efficiency of active flow control in comparison to continuous blowing [14]. An application of active flow control by means of periodic excitation has shown promising effects on our configuration in numerical simulations $[15,16]$.

In this paper we present and discuss the results of a wind tunnel experiment on a nearly full-scale 2.5D model of the pylon-wing junction. The focus of the test campaign lied on the full-scale demonstration of preventing the local separation inboard of the pylon and the suppression thereof by application of aircraft scale AFC actuators on the principle of periodic excitation. A two-stage fluidic system consisting of fluidic amplifiers driven by a fluidic oscillator is employed. It is designed in full-scale, accounting for realistic aircraft constraints. The real-scale experiment is an important step of maturation towards a future flight test. With the employed flow control system, local separation behind the pylon-wing junction can be fully suppressed at Reynolds numbers up to $R e=10.7 \cdot 10^{6}$.

\section{Experimental Setup}

This section introduces the wind tunnel model and the employed flow control system. In addition, the model instrumentation and the corresponding experimental uncertainty is presented.

\section{A. Wind Tunnel Model and Instrumentation}

The experiments were performed using a generic model representing the wing segment of the pylon-wing junction of a reference large transport aircraft. The model consists of a 28 degree swept constant chord wing incorporating the DLR-F15 3eRef [17] three-element airfoil with slat and flap deflected to 28 degrees and 35 degrees, respectively. The high-lift setting corresponds to the designated landing configuration, where achieving maximum lift coefficient is crucial. The wing segment is a moderate down-scale of 3:5 with respect to the reference aircraft used for the investigations within the current project. Special care has been taken to verify that the flow separation behavior of the model geometry is representative for the situation at the full aircraft $[15,16]$.

The wing is equipped with a through-flow nacelle of a corresponding UHBR engine with a diameter of about $1.8 \mathrm{~m}$. The nacelle and pylon geometry, as well as the corresponding nacelle position and slat cutout are at a corresponding scale to the wing to represent the UHBR engine installation of the reference aircraft. The engine nacelle is further equipped with a classical nacelle strake [18] as this is the non-costly standard mean for state-of-the-art underwing-mounted engine installations to mitigate the flow separation risk in this area. The reference chord of the wing is about $3.3 \mathrm{~m}$ and the wing span slightly below $6 \mathrm{~m}$. The low aspect ratio is a tribute to the large size and the allowable weight and aerodynamic loads for the wind tunnel installation. To increase the effective aspect ratio the model is equipped with 

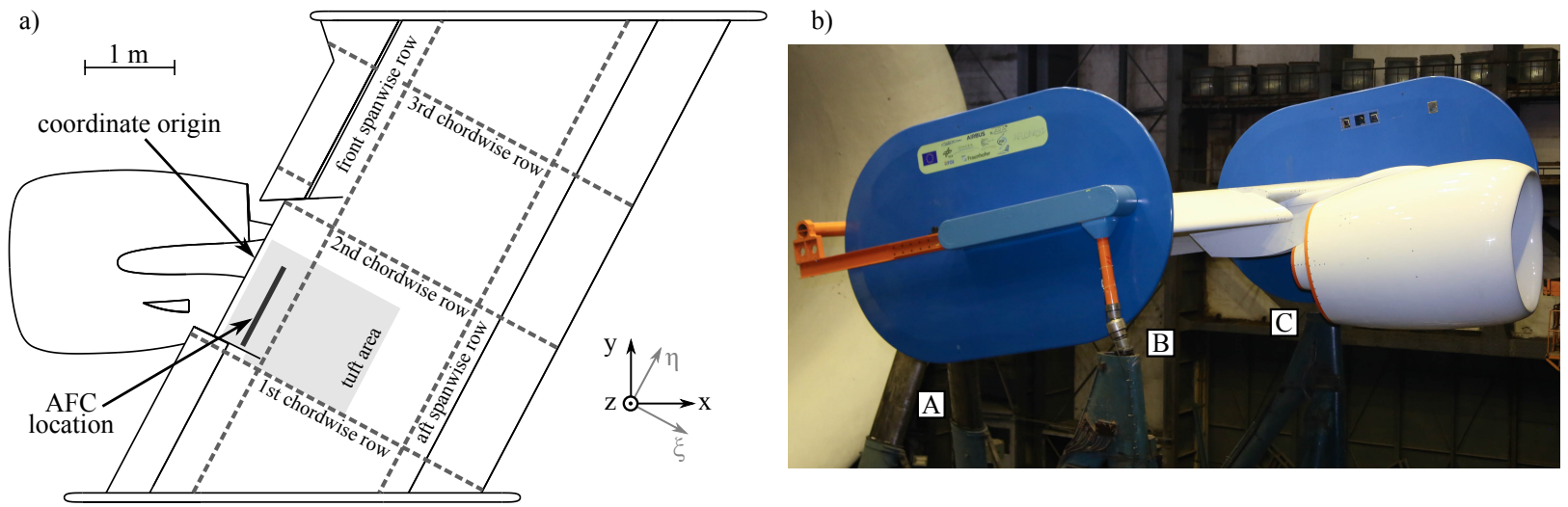

Fig. 1 a) Schematic top view of the wind tunnel model; b) photograph of the wind tunnel model in test section.

side plates. To prevent negative effects at the forward swept outboard side edge, a special leading edge extension has been applied at the slat to prevent a too early wing stall near the side plate. Nevertheless, the impact in terms of lift coefficient is different to the aircraft as the envisaged separation does not trigger the full wing stall as for the real aircraft. Therefore, the lift coefficient can only be used as a relative indicator.

The model is highly instrumented with static pressure readings. In total, 380 pressure taps are distributed over the model in two spanwise and three chordwise rows. Note, that in the scope of this paper, 'chordwise' $\xi$ and 'spanwise' $\eta$ refer to the direction normal and parallel to the wing leading edge respectively. Each chordwise section consists of 110 pressure taps, 25 on the slat, 55 on the wing, and 30 on the flap. Fig. 1a indicates the position of these rows with dashed lines. Spanwise rows consists of 15 to 16 pressure taps depending on the chordwise position.

\section{B. Wind Tunnel and Installation}

The test campaign was conducted at the atmospheric wind tunnel T-101 at the Central Aerohydrodynamic Institute TsAGI (Zhukovsky, Moscow region). The atmospheric wind tunnel has an elliptic cross section of $24 \mathrm{~m} \times 14 \mathrm{~m}$ and allows for an investigation of the model at Reynolds numbers of up to $R e=11 \cdot 10^{6}$ and free-stream Mach numbers of up to $M=0.15$. A six-component electric-mechanical balance measures the forces and moments acting on the model. Mini-tufts are used to visualize the flow in the inboard section of the slat cutout. The section is highlighted with light gray color in Fig. 1. A camera in the inboard side-plate is used to acquire tuft-images at each angle of attack. Fig. 1b shows a photograph of the model during testing. The model is supported by three struts A, B and C. Strut A is used to adjust the angle of attack of the model, while pivoting around points B and C. The struts, beams and side-plates generate parasitic drag. Since the focus is put on increments of the lift performance of the model, this additional drag is not investigated further. 


\section{Active Flow Control System}

The AFC system is located at $10 \%$ relative chord on the inboard side of the slat cutout and covers approximately $50 \%$ of the unprotected leading edge in this area, see Fig. 1. A total of fourteen slots with the dimensions of $60 \mathrm{~mm} \times$ $6 \mathrm{~mm}$ are placed equidistantly in spanwise direction with a spacing of $10 \mathrm{~mm}$. The outlets are inclined at 30 degrees relative to the local surface. These geometrical parameters are recommended by numerical simulations of the wind tunnel model $[15,16]$ and account for the integration constraints of a real aircraft.

The flow control system generates unsteady blowing with a phase-shift of 180 degrees between adjacent outlets by applying the principles of fluidic amplifiers [19] and a fluidic oscillator. In Fig. 2, a schematic representation of the system's internal flow channels is shown. The fluidic oscillator functions as the driving stage of the actuator and generates a self-induced switching between its two branches. Each of these branches is connected to respective pressure ports of the seven fluidic amplifier elements in the outlet stage. The outlet elements consist of bi-stable switches where the main power jet is guided through the respective outlet depending on the control port pressure signal. With the underlying design, no moving parts are required which is a major benefit for the safety-driven aviation industry. A detailed explanation of the actuator and its multi-step design process are published in [20]. Flow control systems applying the same two-stage design were previously successfully tested and published in $[6,8]$.

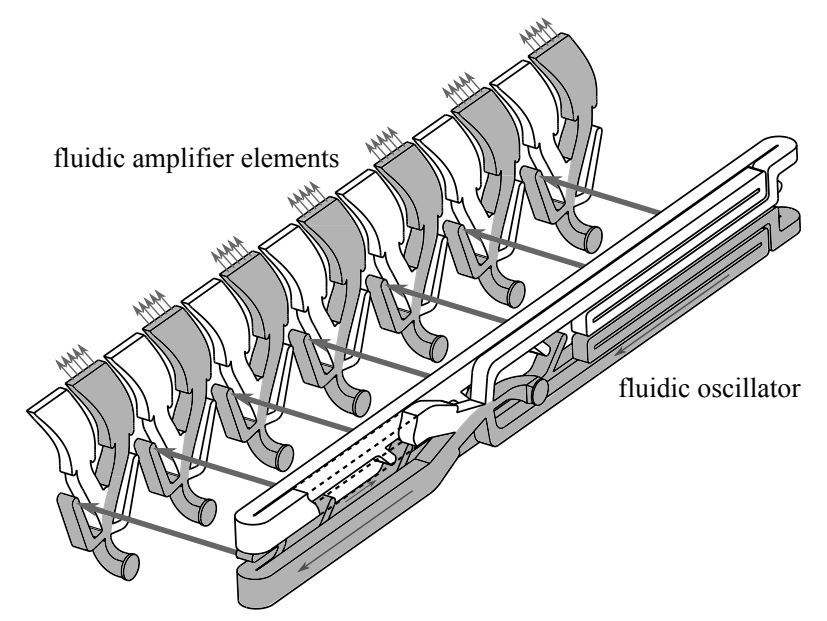

Fig. 2 Schematic of internal flow channels of fluidic AFC actuator; gray area illustrate point in time when flow passes through right outlets.

The flow control actuator is manufactured from milled Aluminum parts for the main fluidic elements and is fitted with 3D-printed inlets. These inlets are connected to the main pressure supply via eight individual corrugated pipes. Details of the actuator design and structural integration can be found in [21]. Bench-top experiments were performed to investigate the function of the system prior to the wind tunnel application. A monitoring system is included in the actuator design which was correlated to mass flow and pressure data during the bench-top experiments. An evaluation of this data is used to select flow control parameters for the wind tunnel test runs. 
Two important AFC parameters, the momentum coefficient $c_{\mu}$ and the velocity ratio $V R$ are defined as follows:

$$
\begin{gathered}
c_{\mu}=\frac{\dot{m} \cdot \bar{u}_{j e t}}{q_{\infty} \cdot S} \\
V R=\frac{u_{j e t, p e a k}}{u_{\infty}} .
\end{gathered}
$$

Here, $q_{\infty}$ is the dynamic pressure, $S$ is the reference area of the wing and $\bar{u}_{j e t}$ is the quadratic mean of the jet velocity.

Figure 3 shows a correlation of the momentum coefficient and the mass flow supplied to the flow control system obtained from bench-top experiments. The derivation of the underlying formulae for the momentum coefficient $c_{\mu}=\operatorname{fun}_{1}\left(\dot{m}, u_{\infty}\right)$ and the velocity ratio $V R=\operatorname{fun}_{2}\left(\dot{m}, u_{\infty}\right)$, both as functions of the mass flow $\dot{m}$ and the free-stream velocity $u_{\infty}$, is detailed in [20]. Thick curves in Fig. 3 represent the resulting behavior at three Reynolds Numbers of interest for the test campaign. Dashed lines represent levels of constant velocity ratio that coincide with levels of constant momentum coefficient at lower mass flows. With the help of these curves, several settings are selected for the wind tunnel tests. Target points are marked with a circle (o) while points evaluated from WT monitoring data are marked with an $\times$ (one $\times$ per angle of attack). Parameters are then chosen to evaluate the influence of AFC with varying $c_{\mu}$ and $V R$ respectively as well as the influence of constant AFC parameters at varying Reynolds numbers.

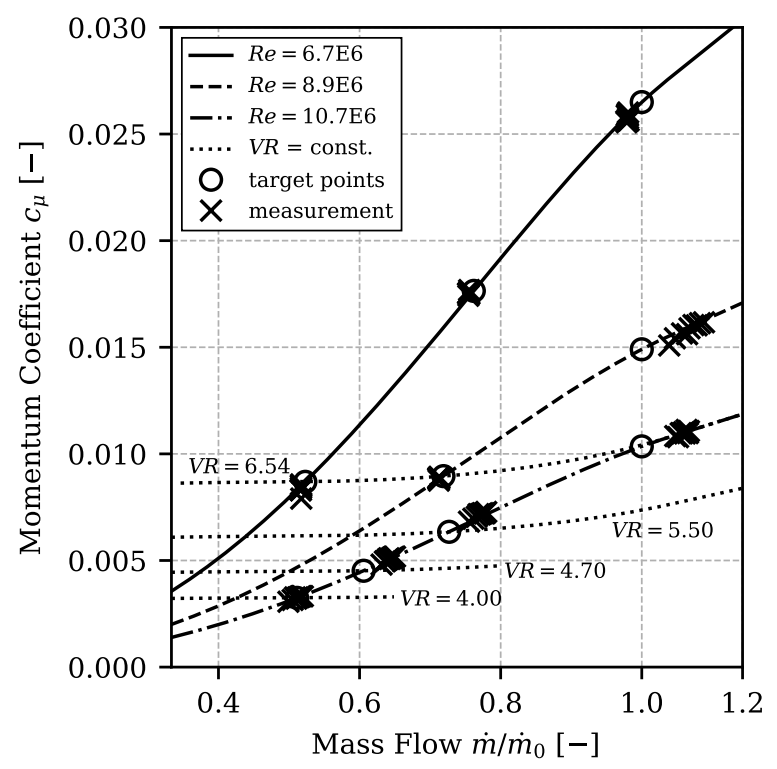

Fig. 3 Evaluation of momentum coefficient at three Reynolds numbers; dotted lines show constant velocity ratios. 


\section{Experimental Uncertainty}

The experimental uncertainty of the lift force measurement is $0.5 \%$ which is equivalent to up to $\Delta C_{L}= \pm 0.015$ at the highest Reynolds number and angle of attack. The accuracy of the pressure sensors in use is stated as $0.1 \%$ of full scale output.The momentum coefficient and velocity ratio are calculated from a correlation of AFC inlet pressure, mass flow and jet Mach number derived during bench-top experiments. Simplifications are taken with respect to the shape of the velocity profile in time and space. A quantification of the resulting error is difficult but since the same correlation is used to calculate all momentum coefficients, a comparison is still valid. The error between the $c_{\mu}$-values is then only determined by the AFC inlet pressure sensors with an accuracy of $\pm 0.125 \%$. This translates to a maximum relative error in $c_{\mu}$ of approximately $\pm 0.25 \%$.

\section{Baseline Flow}

In the following, the baseflow is characterized along the lines of the discussions of lift force data and tuft surface flow visualization. The lift data compares the results for three (chord-based) Reynolds numbers, namely $6.7 \cdot 10^{6}$, $8.9 \cdot 10^{6}$ and $10.7 \cdot 10^{6}$ for the corresponding free-stream Mach numbers of $M=0.09, M=0.12$ and $M=0.14$, respectively. This correlation applies for all results presented within the scope of this paper. Because of the fixed chord length of the model and the nature of the atmospheric wind tunnel, each free-stream Mach number corresponds to one model Reynolds number. For ease of referencing, only the Reynolds number will be named to specify the inflow conditions of a test scenario in the following discussion.

Because of the 2.5D nature of the wing the flow remains attached up to higher incidence angles than it would be the case for a full 3D wing. Flow separation occurs first on the suction side surface of the wing in the region above the pylon, whereas the flow over the remaining wing stays attached. Thus, separation on this model wing is a local effect and does not result in the conventional stall behavior, i.e. the break down of lift and reduction of $C_{L, \max }$, as it would be observed on a finite wing. The effort of active flow control described in the following section will aim at eliminating the separation above the pylon.

Fig. 4 presents the lift coefficient characteristics versus the angle of attack for all three baseline measurements. The angles of attack selected for discussion ( $\alpha_{1}$ to $\alpha_{5}$ ) denote even angles in the measured range where the baseline flow exhibits separated flow, and where additionally $\alpha_{5}$ is the highest angle measured due to limitations of the wind tunnel setup. For angles of attack of up to $\alpha_{1}-1^{\circ}$ the curves coincide for all Reynolds numbers tested. For higher incidences, the unsteadiness of the flow downstream of the wing-pylon junction results in an erratic course of the lift curve for the remaining angles of attack measured. All curves exhibit a kink where the measured lift force drops below the value for linear extrapolation of the lift coefficient gradient for attached flow. This drop in lift coefficient indicates the onset of flow separation in the region behind the slat-cutout, as evidenced by mini-tuft images. The large drop in lift coefficient at $\alpha=\alpha_{5}$ is caused by separation near the outboard side-plate, which is only observed for the lowest Reynolds number 


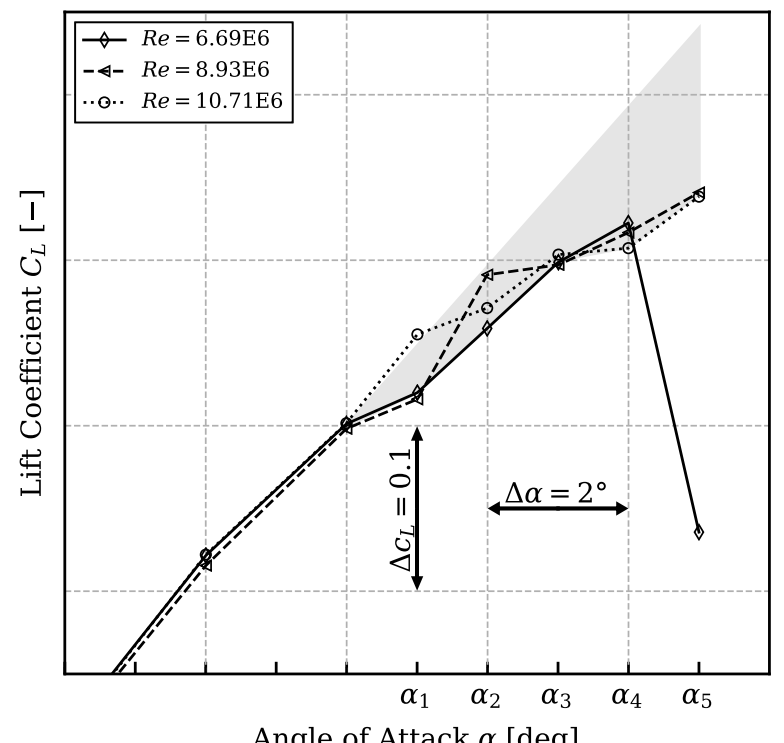

Fig. 4 Lift coefficient versus angle of attack for three baseflows; gray color highlights area of potential AFC effect.

tested. Beside this, the overall $C_{L}$-behavior of the wing segment is independent of Reynolds number effects, as could be expected for Reynolds numbers of approx. $7 \cdot 10^{6}$ and above (cf. [13]).

In Fig. 5, the complex nature of the flow around the pylon-wing junction is represented schematically. The paths of these vortices indicated here are based on previous research of the pylon-wing junction [12] as well as numerical simulations of the configuration [16]. Several vortices are shed from the geometrical features where local changes in the circulation occur, the most dominant vortices being the outboard slat-step (1) and slat vortex (2), the pylon vortex (3), the strake vortex (4), the nacelle vortex, as well as the inboard slat (6) and slat-step vortex (7). Additional upwash is added to already weakened areas behind the unprotected inboard leading edge. The interaction of the longitudinal vortices with the boundary layer further weakens the latter, making the flow more prone to separation. It is important to note that in contrast to engine installations with smaller nacelles the implemented nacelle strake is not able to mitigate the risk of separation on the increased extent of the unprotected leading edge. Outside of the separation region behind the slat cutout, the vortex-induced downwash has a stabilizing effect on the flow. 


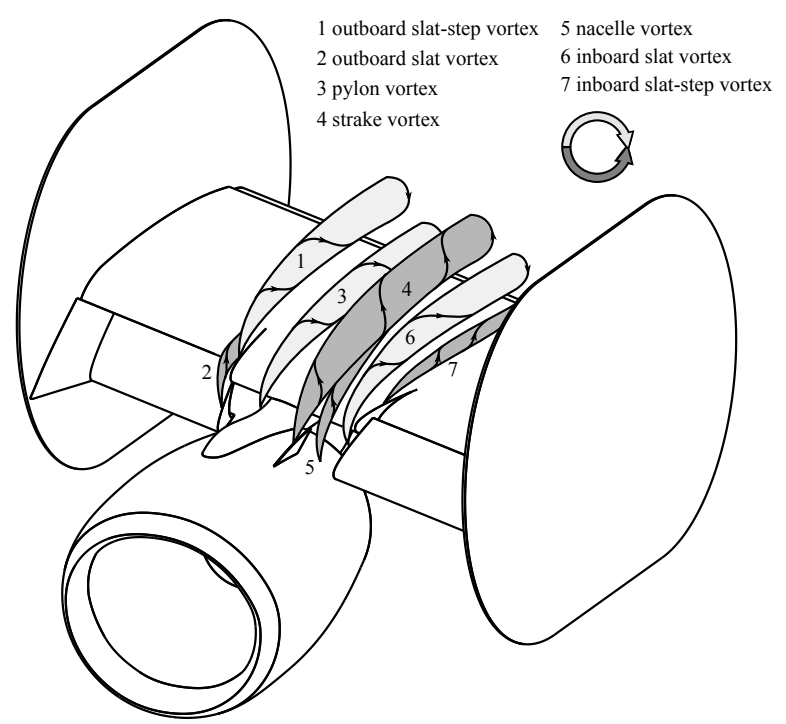

Fig. 5 Schematic depiction of vortex paths around the wind tunnel model (based on $[12,16])$.
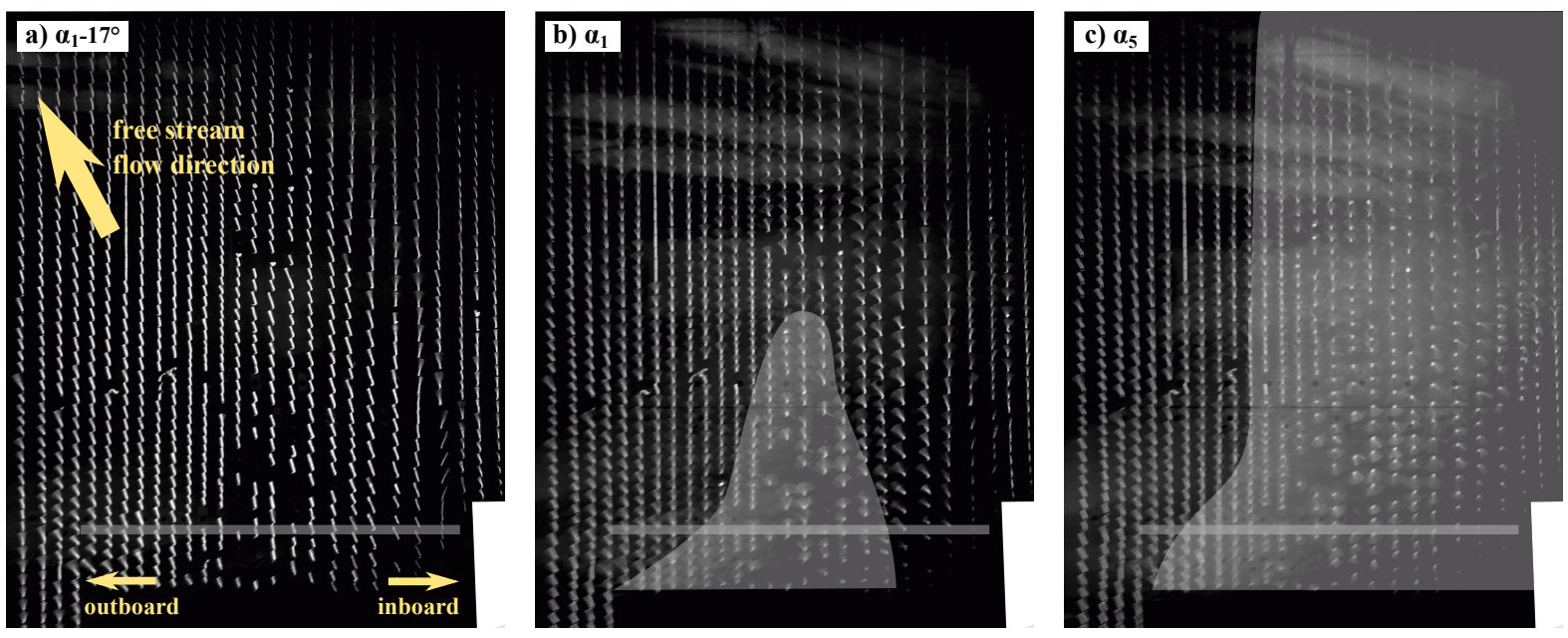

Fig. 6 Tuft flow visualization of baseline flow at $R e=10.7 \cdot 10^{6}$; gray line indicates AFC location, gray areas indicate separation; field of view as identified in Fig. 1a.

The development of the local flow separation can be observed in the tuft image series shown in Fig. 6. For very low incidences (Fig. 6a) the flow is fully attached. The surface flow, as indicated by the orientation of the tufts, is aligned with the main flow direction of the oncoming flow and exhibits no unsteadiness. Only in the region of the footprint of the slat-step vortex a higher unsteadiness of the tufts is observed, and the mean flow direction is normal to the leading edge due to the rotational sense of the slat-step vortex and the acceleration through the slat gap. With increasing incidence, the flow starts to separate at the wing in the region immediately downstream the inboard side of the wing-pylon junction (see gray area in Fig. 6b). However, despite strong cross-flow and high unsteadiness, the flow 
remains attached on the majority of the wing segment shown, especially at the outboard side of the wing-pylon junction. Further increase of the angle of attack results in the enlargement of the region of separated flow until it reaches the downstream border of the field of view of the tufts and possibly extending until the trailing edge of the wing segment (see Fig. 6c).

Fig. 7 shows the pressure distributions along the two spanwise lines of pressure taps on the main wing element at the angle of attack $\alpha=\alpha_{4}$, i.e. before side-plate separation sets in for the measurement at $\operatorname{Re}=6.7 \cdot 10^{6}$. The origin of the spanwise coordinate is at the intersection of the wing leading edge with the center line of the pylon, see Fig. 1. The measurements at the three different speeds show only a marginal deviation from each other close to the uncertainty of the pressure measurements. The measurements of the front row exhibit increased pressure coefficients between $-0.15<\eta<0.15$. These values correspond to the pressure taps in the segment behind the nacelle and pylon, where no slat is installed. Inboard and outboard the slat cutout, pressure levels of the front row decrease, indicating lower pressure on the leading edge due to the slat. Fig. 8 shows the pressure distribution of the first chordwise row which is located just inboard of the slat cutout, see Fig. 1a. Full agreement of the pressure coefficients can be noted for the slat and the main element, whereas neglectable differences are visible at the suction peak of the flap. Overall, the pressure distributions presented in Fig. 7 and 8 show similar flow topologies behind and inboard of the slat cutout for all three baseflow measurements, indicating no significant Reynolds number effect within the measured range.

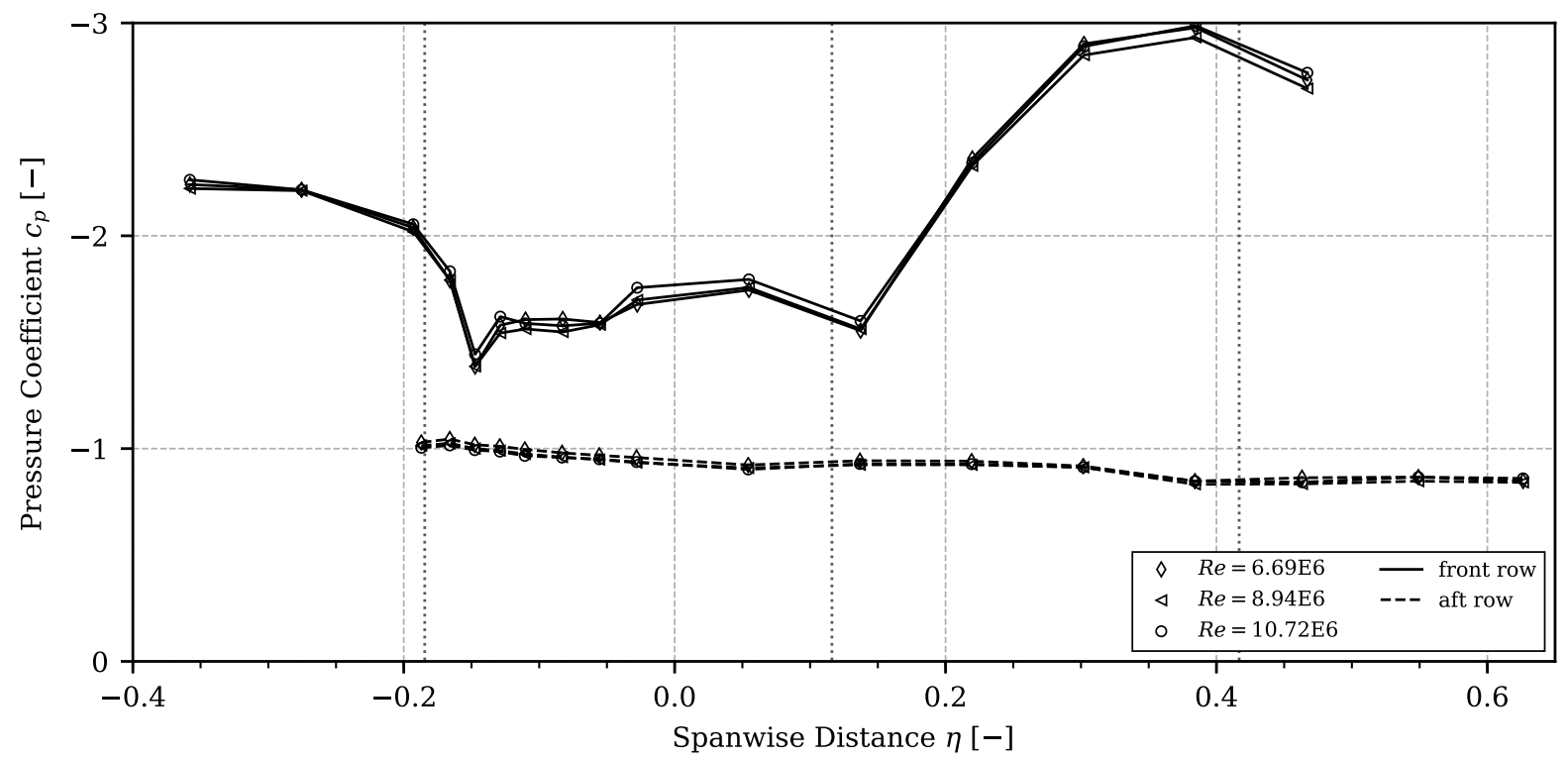

Fig. 7 Spanwise pressure distribution for all baseflows at $\alpha=\alpha_{4}$; dotted vertical lines indicate position of chordwise pressure taps. 


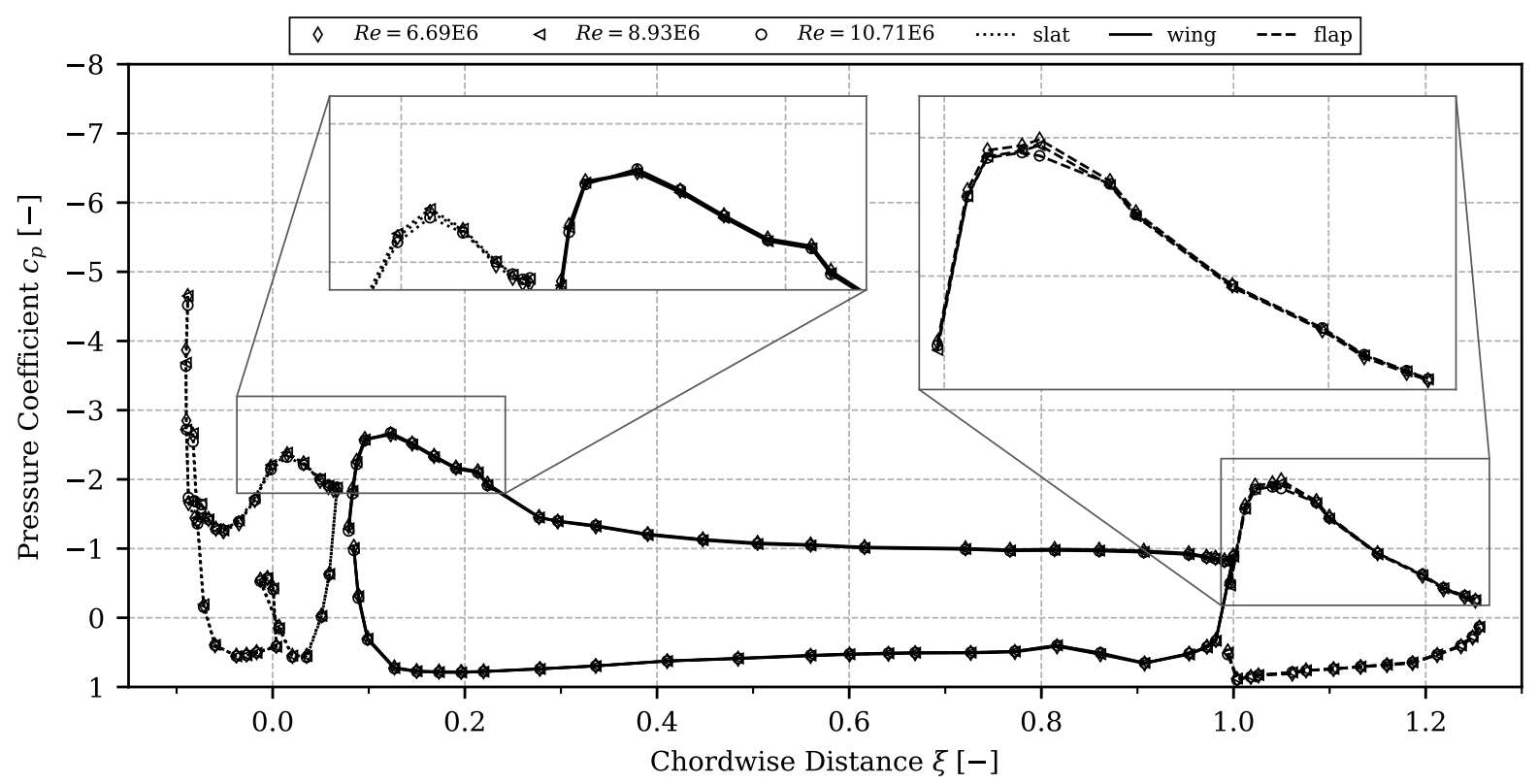

Fig. 8 Chordwise pressure distribution ( $1^{\text {st }}$ row) for all baseflows at $\alpha=\alpha_{4}$.

\section{Actuated Flow}

In this section, results of the actuated flow investigation are presented. The analysis focuses on the global effect of AFC on the lift coefficient. Pressure data facilitates the identification of the underlying flow mechanisms. The first subsection discusses the influence of a variation of the momentum coefficient and velocity ratio at the highest Mach and Reynolds number. The second subsection discusses results at different Reynolds numbers, where the pure momentum coefficient effect can by investigated at a constant velocity ratio.

\section{A. Influence of Flow Control Parameter Variation at constant Reynolds number}

In this section we focus on the effect of a variation of $c_{\mu}$ and $V R$ at the highest Reynolds number of $R e=10.7 \cdot 10^{6}$. Four different parameter settings in the range of $0.32 \% \leq c_{\mu} \leq 1.08 \%$ and $4.0 \leq V R \leq 6.5$ are investigated.

The resulting lift curves of the baseline and the four actuated flows are shown in Fig. 9. Although a full stall of the wind tunnel model was not reached at the maximum possible angle of attack $\alpha_{5}$, a distinct influence of the AFC system is visible in the global force coefficients. Especially at the angles of attack labeled with $\alpha_{1}$ to $\alpha_{5}$, the application of active flow control yields an increase in $C_{L}$ of up to 7 lift counts. Even more important, the application of active flow control extends the linear range of the lift curve and removes the 'kink' in the lift curve of the base flow indicating the local flow separation. In addition, a clear offset of up to 4 lift counts is achieved in the linear part of the lift curve $\left(\alpha<\alpha_{1}\right)$. A clear trend of the local AFC effect is visible especially at $\alpha=\alpha_{3}$. With increasing momentum coefficient, the lift increases further. No saturation is reached with respect to the lift increase within the measured range of momentum coefficients. 


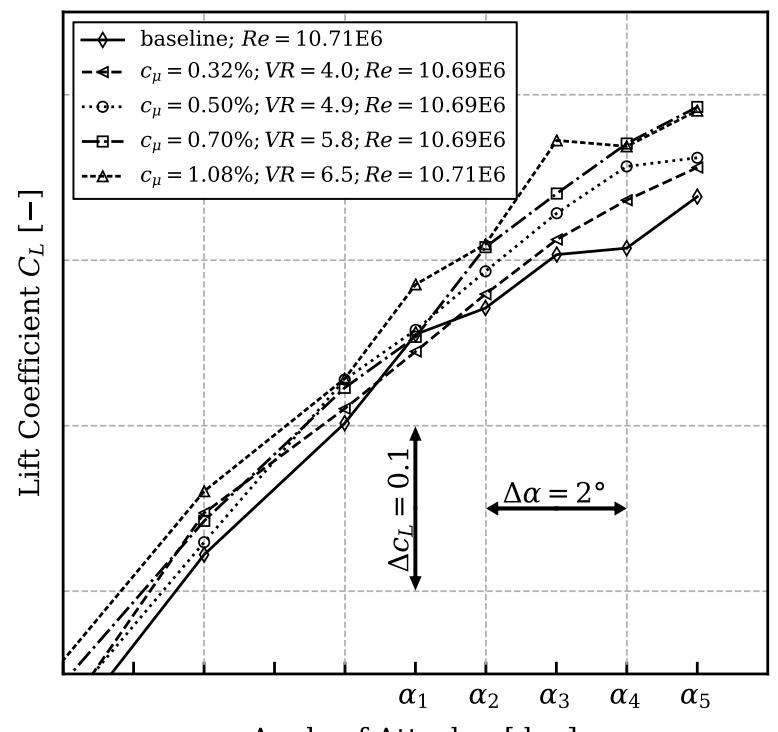

Angle of Attack $\alpha$ [deg]

Fig. 9 Lift coefficient versus angle of attack at $R e=10.7 \cdot 10^{6}$.

In Fig. 10 the spanwise pressure distribution at $\alpha=\alpha_{5}$ is presented for the baseline flow and two measurements with actuation $\left(c_{\mu}=0.70 \%\right.$ and $\left.1.08 \%\right)$. Solid lines correspond to the front spanwise row of pressure taps close to the leading edge, whereas the dashed lines correspond to the aft row close to the trailing edge of the main element (see Fig. 1). Major differences between the actuated and non-actuated cases can be noted between $-0.15<\eta<0.0$. These values correspond to the pressure taps downstream of the AFC location. The pressure coefficients of both measurements with actuation coincide but reach higher values than for the baseline flow. With actuation, the flow downstream of the AFC location reattaches, thereby restoring the suction peak on the unprotected leading edge. In turn, the pressure gradient increases. The stronger recompression leads to high pressure coefficients at the location of the first spanwise measurement row, as depicted in Fig. 10. Fig. 11 shows the pressure distribution of the first chordwise row which underlines the interpretation of the spanwise pressure distribution. The higher suction peak at the leading edge due to the effective AFC application causes higher pressure gradients between the AFC system at $\xi \approx 0.12$ and the spanwise pressure taps at $\xi \approx 0.2$ leading to the increased pressure coefficients at the first spanwise row, as noted above. Since the first chordwise pressure tap row is inboards of the sensitive area, it does not show the pressure rise at the actuation location itself.

The local effect of AFC on the sectional lift can be estimated by integrating the pressure distribution. Applying $c_{\mu}=0.70 \%$ increases the sectional lift coefficient in the first chordwise row by $7 \%$. Increasing the momentum coefficient by a factor of 1.5 to $c_{\mu}=1.08 \%$ further improves the sectional lift coefficient by an additional $1.5 \%$ to a total local gain of $8.3 \%$ with respect to the baseline. 


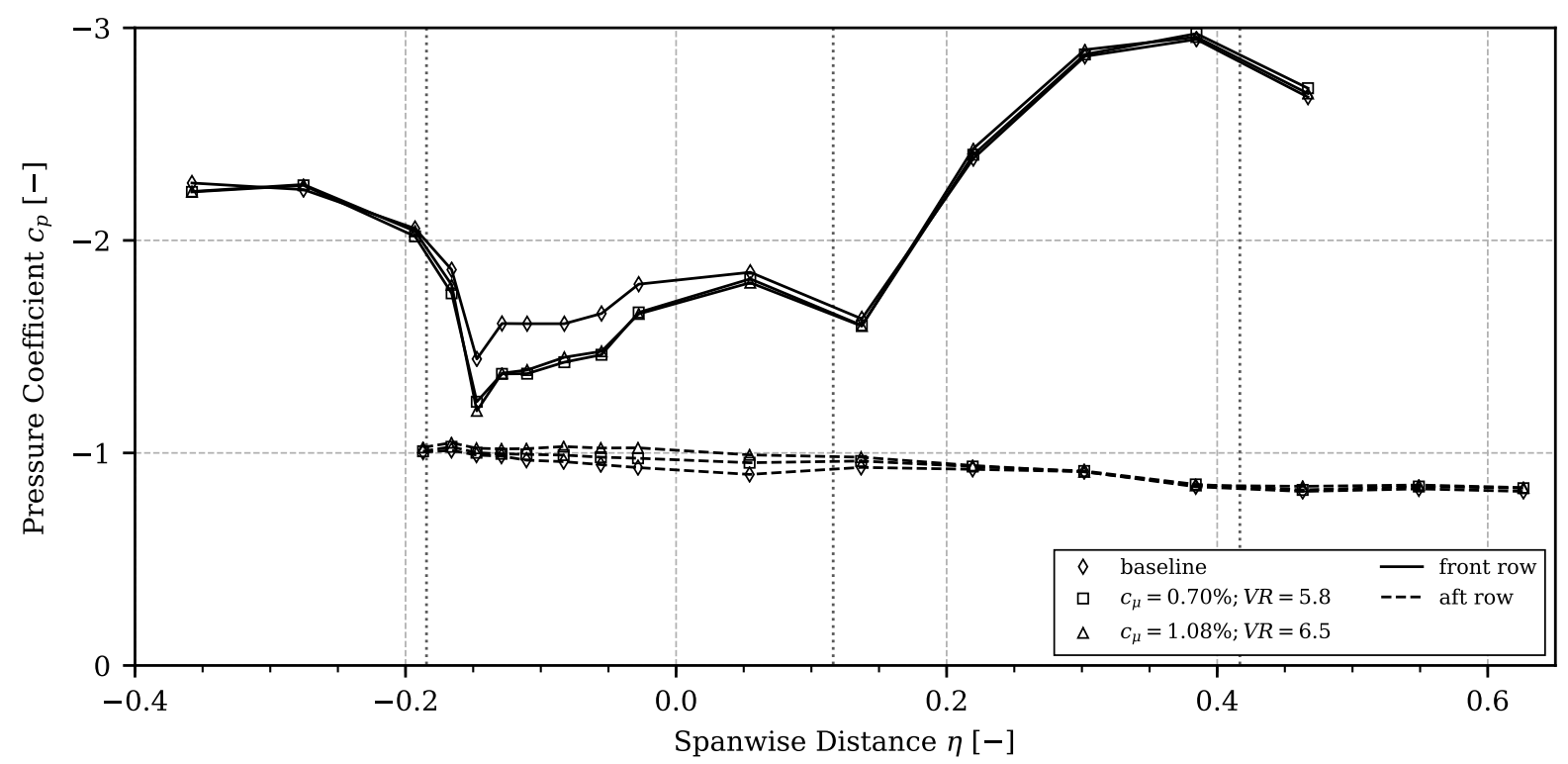

Fig. 10 Spanwise pressure distribution for baseline flow and two actuated flows at $\operatorname{Re}=10.7 \cdot 10^{6}$ and $\alpha=\alpha_{5}$; dotted vertical lines indicate position of chordwise pressure taps.

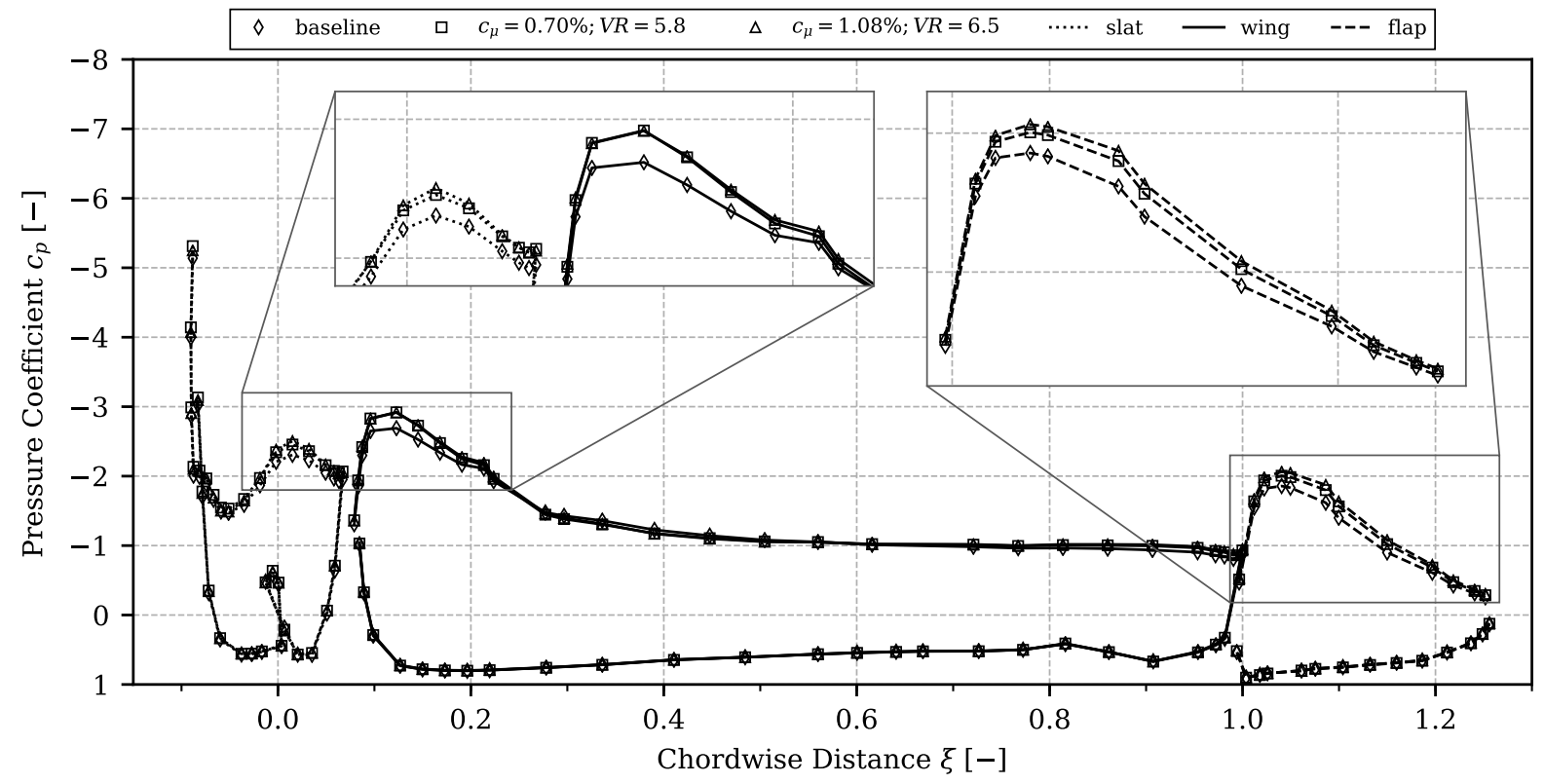

Fig. 11 Chordwise pressure distribution $\left(1^{\text {st }}\right.$ row $)$ for baseline flow and two actuated flows at $\operatorname{Re}=10.7 \cdot 10^{6}$ and $\alpha=\alpha_{5}$. 
Returning to the spanwise pressure distribution in Fig. 10, the pressure measurements at the aft row show a distinct difference downstream of the AFC location at $\eta=0.0$. For the baseline flow, the pressures are higher than for the actuated cases, indicating an extension of the separated flow area up to the trailing edge of the main element. The chordwise pressure distribution in Fig. 12 further emphasizes this difference. Whereas already evident on the main element at $\xi>0.7$, the varying pressure distribution is even more pronounced on the flap. If we estimate the local effect of AFC at the pressure tap row downstream of the actuated region by integrating the pressure distribution on the flap, we calculate an increase of $13 \%$ in sectional lift coefficient by applying $c_{\mu}=0.70 \%$. An increase of momentum coefficient to $c_{\mu}=1.08 \%$ yields a further gain of $4 \%$ sectional lift coefficient. The pressure distributions in Fig. 10,11 and 12 indicate that the higher momentum coefficient and corresponding velocity ratio have a stronger influence on the flow around the model elements downstream of the AFC location. We conclude from this data that no saturation is reached within the investigated range of momentum coefficients, although similar $C_{L}$-values are measured at $\alpha=\alpha_{5}$.

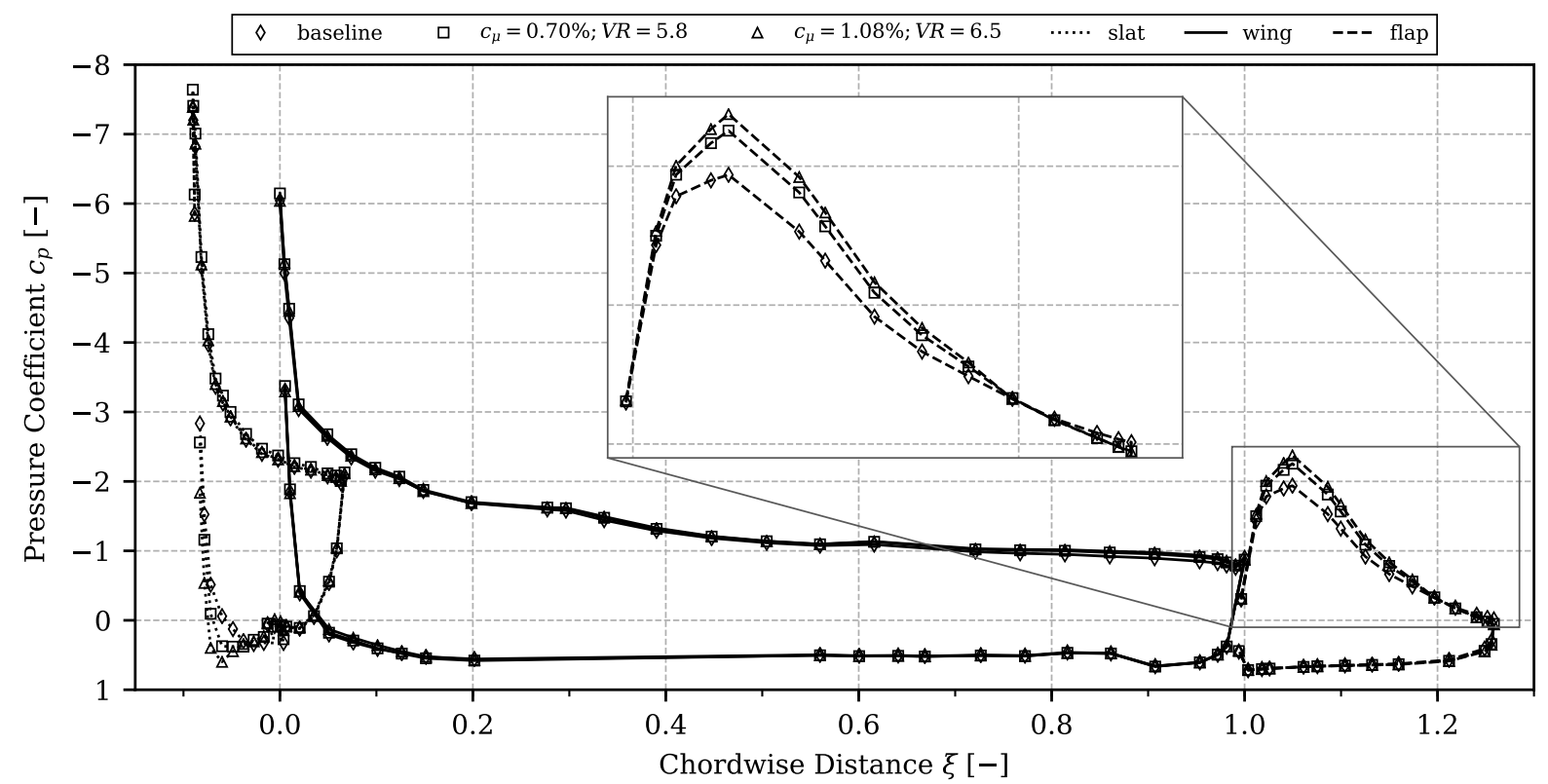

Fig. 12 Chordwise pressure distribution ( $2^{\text {nd }}$ row) for baseline flow and two actuated flows at $\operatorname{Re}=10.7 \cdot 10^{6}$ and $\alpha=\alpha_{5}$. 
a) baseline; $\operatorname{Re}=10.7 E 6 ; \alpha=\alpha_{5}$

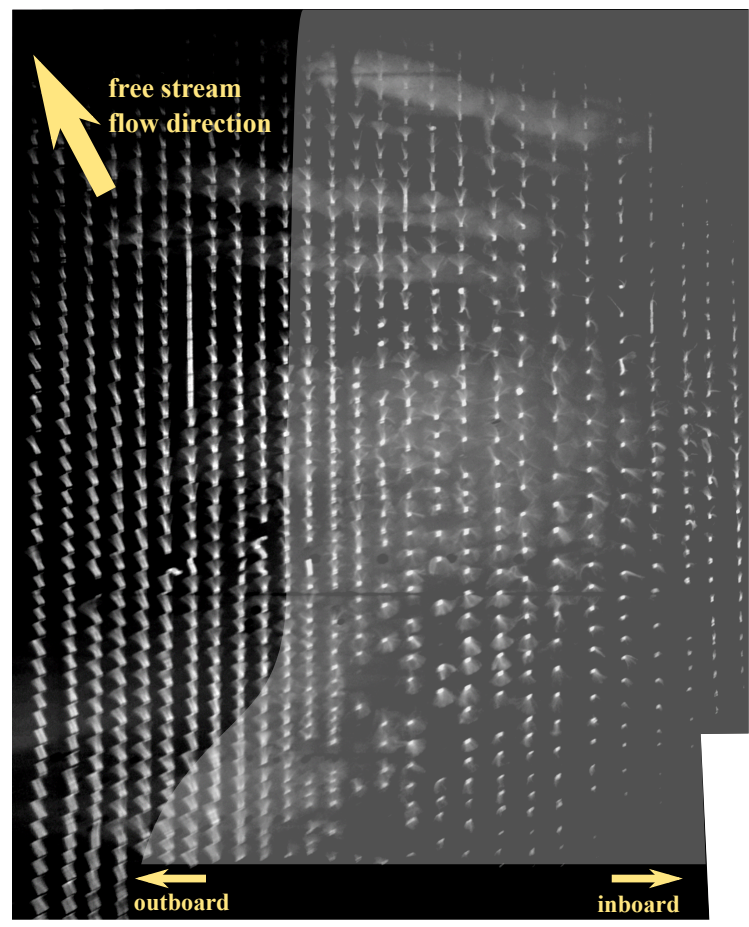

b) $c_{\mu}=1.08 \% ; \operatorname{Re}=10.7 \mathrm{E} 6 ; \alpha=\alpha_{5}$

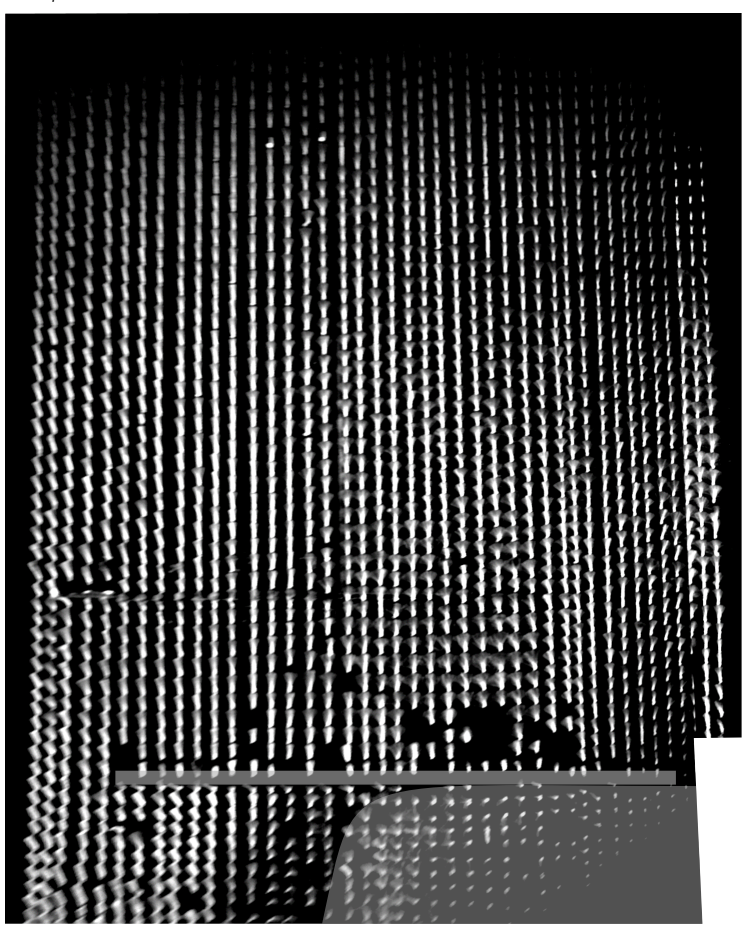

Fig. 13 Tuft flow visualization at $\alpha=\alpha_{5}$ and $\operatorname{Re}=10.7 \cdot 10^{6}:$ a) baseline flow b) actuated flow with $\mathrm{c}_{\mu}=1.08 \%$; gray line indicates AFC location, gray areas indicate separation; field of view as identified in Fig. 1a.

The change in the local flow separation behind the slat cutout can be observed in the comparison of two tuft flow visualizations in Fig. 13. A visualization of the baseline flow at $R e=10.7 \cdot 10^{6}$ and $\alpha=\alpha_{5}$ is revisited in Fig. 13a to allow for an easier comparison with the corresponding actuated flow in Fig. 13b. The baseline shows significant separation in the area trailing the slat cutout between the pylon and inboard slat. In the area downstream of the slat side-edge, the separation spreads across the whole length of the field of view. When controlling the flow with $c_{\mu}=1.08 \%$, minor separation is still present close to the leading edge and the slat side-edge, but the large separated area downstream of the AFC location is eliminated, see Fig. 13b. In contrast to the attached base flow at low incidence, cf. Fig. 6a, the tufts trailing the AFC location are oriented normal to leading edge, thereby showing a clear influence of the emanating jets. Tufts on the outboard side of the field of view (left side of Fig. 13b) are gradually oriented more in the direction of the free-stream flow, indicating lower response to the flow control effort. In the inboard region trailing the slat side-edge, the tufts are oriented in an inboard direction. This region is heavily influenced by vortices shed from the slat side-edge and the leading edge slat-step and shows strong unsteadiness in the tuft visualization. The application of AFC stabilizes this region and no separation occurs. 


\section{B. Influence of Flow Control Parameters on AFC Effect across Wind Tunnel Speeds}

The focus of the test campaign lies on the investigation of the flow at the highest Reynolds number of $R e=10.7 \cdot 10^{6}$. The corresponding Mach number of $M=0.14$ limits the examinable flow control parameters for the actuator system at hand to $c_{\mu}<1.08 \%$ and $V R<6.5$. With further investigations at different wind tunnel speeds, resulting in lower free-stream Mach and Reynolds numbers, the parameter space can be extended up to $c_{\mu} \approx 2.5 \%$ and $V R \approx 10$.

Three measurements are compared to analyze the effect of active flow control across different Reynolds numbers and corresponding Mach numbers. The same AFC parameters are selected for all three cases. If we recall Fig. 3, the highest possible $c_{\mu}$ at $R e=10.7 \cdot 10^{6}$ obtains the same $V R$ as the measurement at the lowest $c_{\mu}$ at $R e=6.7 \cdot 10^{6}$. Equivalent parameters are measured at the intermediate Reynolds number of 8.9 million to obtain the same VR. Fig. 14 shows the lift curves of these three measurements as well as the corresponding baselines. All curves corresponding to actuated flow show a clear offset in the linear regime below $\alpha_{1}$. The linear portion of the lift curve extends further than in the baseline measurements, i.e. the kinks in the baselines caused by local separation are recovered at $\alpha_{1}$ and $\alpha_{2}$ respectively. For the two measurements at $\operatorname{Re}=6.7 \cdot 10^{6}$, the lift drop at $\alpha=\alpha_{5}$ is caused by side-plate separation and should not be taken into account. A further direct comparison of the global coefficients at $\alpha>\alpha_{1}$ is difficult due to the unsteady nature of the local flow separation. The pressure distributions allow for a better comparison of the local effects.

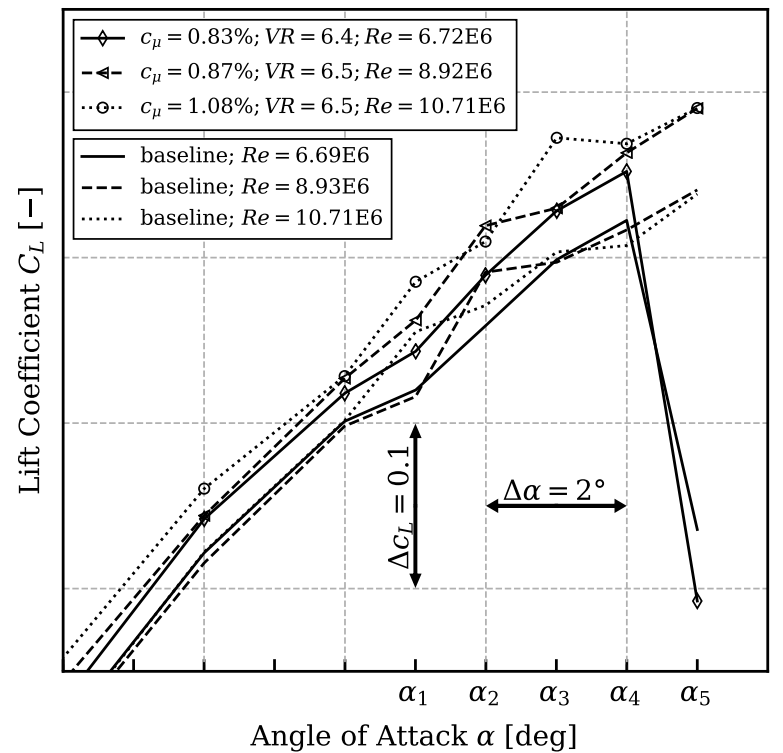

Fig. 14 Lift coefficient versus angle of attack at constant velocity ratio $V R \approx 6.5$. 
In Fig. 15 the spanwise pressure distribution is plotted for three measurements at constant velocity ratio of $V R=6.5$. The data is evaluated at $\alpha=\alpha_{4}$, so side plate separation has not yet set in for the measurement at $R e=6.7 \cdot 10^{6}$. The resulting global forces are similar for all three measurements $( \pm 0.3 \%$ of the average value $)$ as can be seen in the lift curves in Fig. 14. The three pressure distributions coincide across the whole span with the exception of the segment behind the AFC location at $-0.15<\eta<-0.02$. Minor differences are further visible in the aft row close to the inboard side-plate. The similarity of the pressure distributions and with that the flow topologies is further emphasized by Fig. 16. Here, the pressure distributions are shown for the second chordwise row at the angle of attack $\alpha_{4}$. Curves of the slat, main element and flap coincide very well for all measurements across the tested Reynolds and corresponding Mach numbers. While there are very small differences in the local pressure distribution downstream of the AFC location, it is apparent that the resulting overall topologies are similar. We conclude that in this case of active flow control, the velocity ratio is the governing parameter for AFC since at the same velocity ratio, the impact of varying momentum coefficient is neglectable regardless of the Reynolds number and the free-stream Mach number.

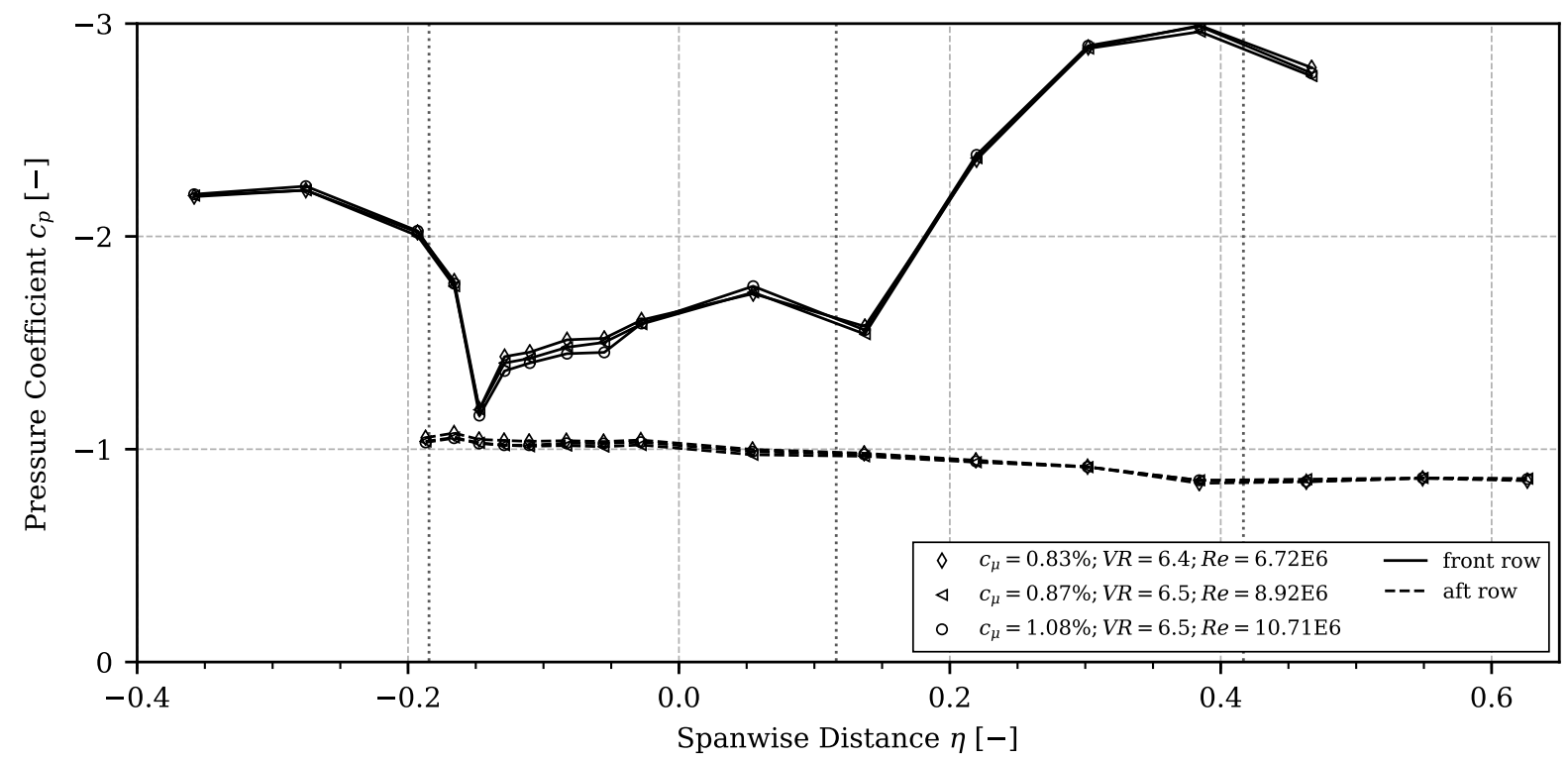

Fig. 15 Spanwise pressure distribution for three actuated flows at $\mathrm{VR}=6.5$ and $\alpha=\alpha_{4}$; dotted vertical lines indicate position of chordwise pressure taps. 


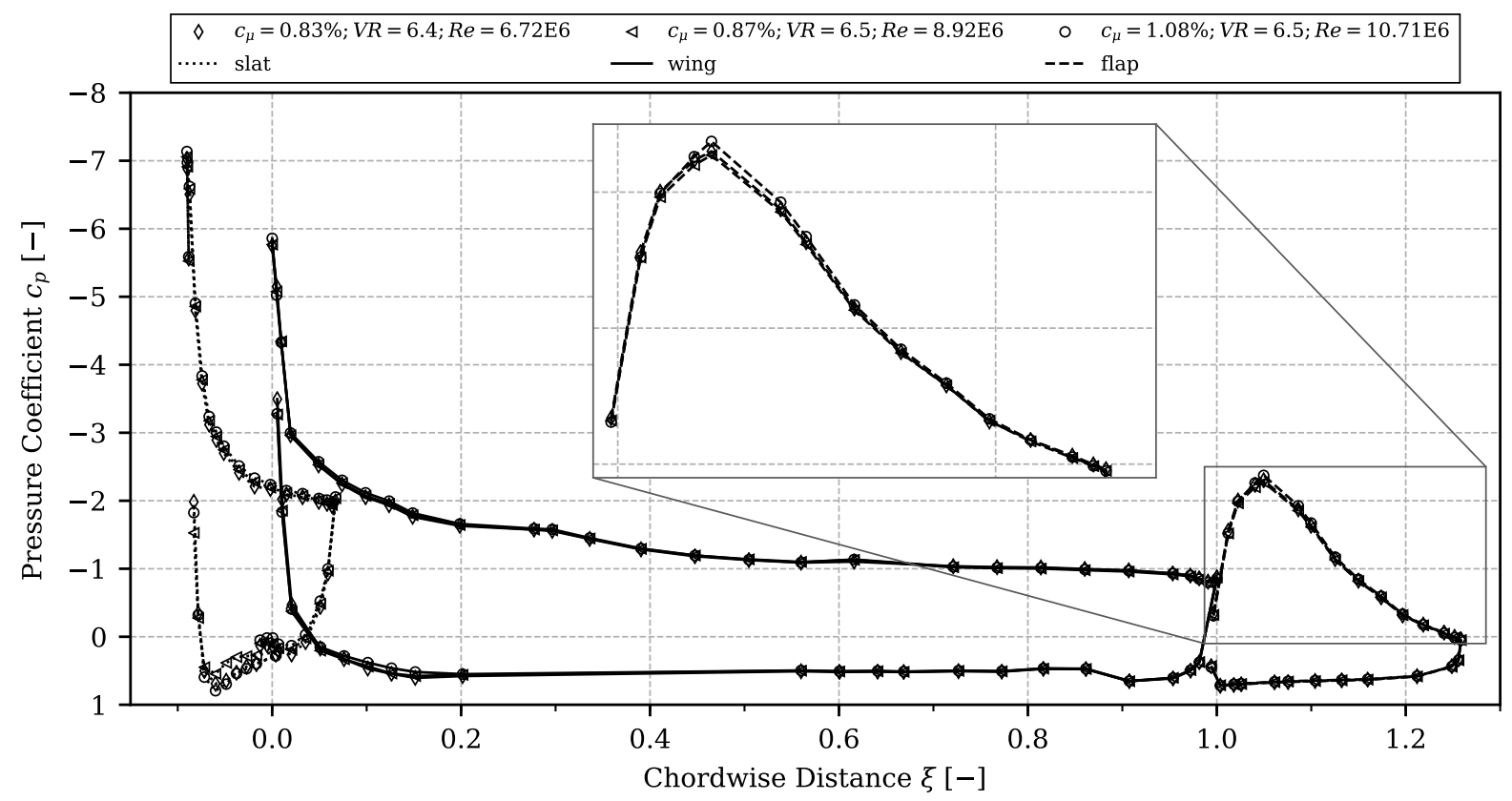

Fig. 16 Chordwise pressure distribution ( $2^{\text {nd }}$ row) for baseline flow and three actuated flows at VR $=6.5$ and $\alpha=\alpha_{4}$.

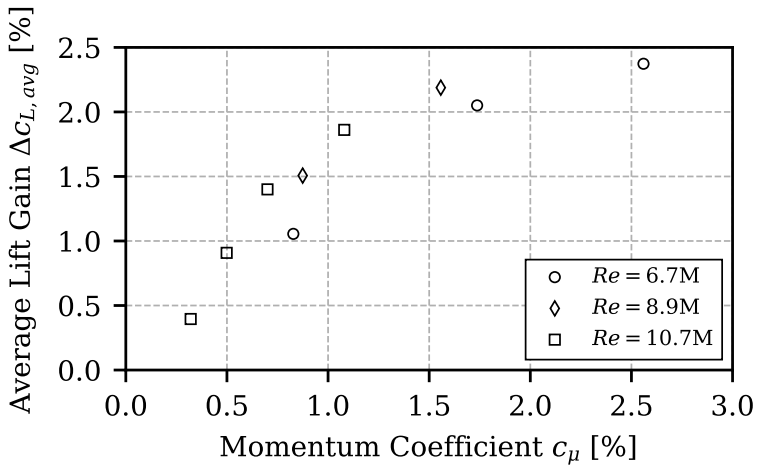

Fig. 17 Average relative increase in lift coefficient between $\alpha_{1}$ and $\alpha_{5}$.

Fig. 17 summarizes the relative lift gain for all measurements across all Reynolds numbers tested. Since no maximum lift and angle of attack could be investigated, the increase in lift $\Delta C_{L}$ is averaged over angles of attack $\alpha_{1}$ to $\alpha_{5}$. The relative values are calculated with respect to their respective baseline values. Angles of attack where side plate separation occurs are omitted from the analysis. The data shows a clear trend of higher $\Delta C_{L}$ with increasing momentum coefficients for all Reynolds numbers. At higher momentum coefficients the slope $\frac{\partial\left(\Delta C_{L}\right)}{\partial c_{\mu}}$ of the overall trend is reduced. Thus, the additional gain in lift per momentum invested decreases. This commonly indicates a change in the mode of flow control from separation control to circulation control [22]. Once the control authority is sufficiently high to fully reattach the flow, any increase in the forcing amplitude will only result in the less efficient modification of 
a healthy flow, e.g. the reduction of the local spanwise flow component.

The trends of the lift increase of the measurements at $R e=8.9 \cdot 10^{6}$ and $R e=10.7 \cdot 10^{6}$ coincide, whereas the trend of $R e=6.7 \cdot 10^{6}$ is offset by approximately $-0.5 \%$. This offset is not related to a Reynolds or Mach number dependence of the AFC effect, but rather to the sensitive region near the outboard side-plate. For the highest Reynolds number at a Mach number of $M=0.14$, the lift gain increases almost linearly with increasing momentum coefficient and no reduction of slope in this correlation is observed in the tested $c_{\mu}$-range. The average lift gain is approximately $2 \%$ for the maximum actuation amplitude of $c_{\mu}=1.08 \%$.

\section{Conclusion}

Wind tunnel experiments were conducted to investigate the effect of Active Flow Control (AFC) on local flow separation behind the pylon-wing junction of a 2.5D model fitted with an ultra-high-bypass-ratio nacelle. A 3:5-scale model allows for the investigation at realistic Reynolds and Mach numbers up to $10.7 \cdot 10^{6}$ and 0.15 respectively. AFC by means of periodic excitation is employed by a real-scale AFC system. Pulsed jets are generated by applying the principles of fluidic amplifiers and oscillators.

The experiment has demonstrated that local flow separation behind the slat cutout can be fully eliminated by applying AFC. Pressure data and surface flow visualization with mini-tufts underline this conclusion. On the global scale, an application of $c_{\mu}=1.08 \%$ results in a lift gain of approximately $2 \%$ at $R e=10.7 \cdot 10^{6}$. No saturation of the AFC effect is found within the range of investigated $c_{\mu}$-values. A comparison of the flow at constant velocity ratio and three different Mach numbers and corresponding Reynolds numbers shows that the local separation and AFC application is independent of Reynolds number and Mach number effects in the range of $6.7 \cdot 10^{6} \leq R e \leq 10.7 \cdot 10^{6}$ and $0.09 \leq M \leq 0.14$ respectively.

The successful demonstration of a real-scale flow control system paves the way for future flight testing of AFC at the pylon-wing junction.

\section{Acknowledgments}

The work described in this paper and the research leading to these results have received funding from the European Community's Seventh Framework Programme FP7/2007-2013, under grant agreement no 604013, AFLONEXT project. Special thanks is extended to Anna Gebhardt and Vlad Ciobaca, DLR Braunschweig, for their support during the test campaign. Further thanks is extended to the TsAGI T-101 wind tunnel crew as well as all participants of work package 2.1 of the AFLONEXT project. 


\section{References}

[1] Haines, A., Scale Effects on Aircraft and Weapon Aerodynamics, AGARD-AG-323, 1994.

[2] Rudnik, R., and Geyr, H., “The European High Lift Project EUROLIFT II - Objectives, Approach, and Structure,” 25th AIAA Applied Aerodynamics Conference, AIAA Paper 2007-4296, 2007.

[3] Geyr, H., Schade, N., van der Burg, J., Eliasson, P., and Esquieu, S., "CFD Prediciton of Maximum Lift Effects on Realistic High-Lift-Commercial-Aircraft-Configurations within the European project EUROLIFT II," 25th AIAA Applied Aerodynamics Conference, AIAA Paper 2007-4299, 2007.

[4] Rudnik, R., "Stall Behaviour of the EUROLIFT High Lift Configurations," 46th AIAA Aerospace Sciences Meeting and Exhibit, AIAA Paper 2008-836, 2008.

[5] Kato, H., Watanabe, S., Murayama, M., Yokokawa, Y., and Ito, T., "PIV Investigation of Nacelle Chine Effects on High-Lift System Performance," 46th AIAA Aerospace Sciences Meeting and Exhibit, AIAA Paper 2008-240, 2008.

[6] Bauer, M., Lohse, J., Haucke, F., and Nitsche, W., "High-Lift Performance Investigation of a Two-Element Configuration with a Two-Stage Actuator System,” AIAA journal, Vol. 52, No. 6, 2014, pp. 1307-1313.

[7] Pack Melton, L. G., Koklu, M., Andino, M. Y., Lin, J. C., and Shellabarger, E., “Active Flow Control for Trailing Edge Flap Separation,” 2018 AIAA Aerospace Sciences Meeting, AIAA Paper 2018-1799, 2018.

[8] Bauer, M., Grund, T., and Nitsche, W., "Experiments on active drag reduction on a complex outer wing model," AIAA Journal, Vol. 53, No. 7, 2014, pp. 1774-1783.

[9] Ciobaca, V., Wild, J., Bauer, M., Grund, T., Huehne, C.-P., Scholz, P., and Stefes, B., "Wind tunnel experiments with active flow control for an outer wing model," 33rd AIAA Applied Aerodynamics Conference, AIAA Paper 2015-2728, 2015.

[10] Whalen, E. A., Lacy, D. S., Lin, J. C., Andino, M. Y., Washburn, A. E., Graff, E. C., and Wygnanski, I. J., "Performance Enhancement of a Full-Scale Vertical Tail Model Equipped with Active Flow Control,” 53rd AIAA Aerospace Sciences Meeting, AIAA Paper 2015-0784, 2015.

[11] Lin, J. C., Andino, M. Y., Alexander, M. G., Whalen, E. A., Spoor, M. A., Tran, J. T., and Wygnanski, I. J., "An Overview of Active Flow Control Enhanced Vertical Tail Technology Development," 54th AIAA Aerospace Sciences Meeting, AIAA Paper 2016-0056, 2016.

[12] Lengers, M., "Industrial Assessment of Overall Aircraft Driven Local Active Flow Control," Proceedings of the 29th Congress of the International Council of the Aeronautical Sciences, ICAS Paper 2014-0175, 2014.

[13] Rudnik, R., and Germain, E., "Re-No. Scaling Effects on the EUROLIFT High Lift Configurations," 45th AIAA Aerospace Sciences Meeting and Exhibit, AIAA Paper 2007-752, 2007.

[14] Seifert, A., Bachar, T., Koss, D., Shepshelovich, M., and Wygnanskil, I., "Oscillatory Blowing: A Tool to Delay Boundary-Layer Separation,” AIAA Journal, Vol. 31, No. 11, 1993, pp. 2052-2060. 
[15] Fricke, S., Ciobaca, V., Kröhnert, A., Wild, J., and Blesbois, O., “Active Flow Control Applied at the Engine-Wing Junction,” 5th CEAS Air and Space Conference, CEAS 2015 Paper No. 249.

[16] Fricke, S., Ciobaca, V., Wild, J., and Norman, D., "Numerical Studies of Active Flow Control Applied at the Engine-Wing Junction," Advances in Simulation of Wing and Nacelle Stall, Notes on Numerical Fluid Mechanics and Multidisciplinary Design, Vol. 131, Springer International Publishing, 2016, pp. 397-411.

[17] Wild, J., "Mach and Reynolds number dependencies of the stall behavior of high-lift wing-sections," Journal of Aircraft, Vol. 50, No. 4, 2013, pp. 1202-1216.

[18] Schaufele, R., "Applied Aerodynamics at the Douglas Aircraft Company - A Historical Perspective," 37th Aerospace Sciences Meeting and Exhibit, AIAA Paper 99-0118, 1999.

[19] Kirshner, J. M., “Fluid Amplifiers,”, 1966.

[20] Schlösser, P., and Bauer, M., "Design of a Pulsed Jet Actuator for Separation Control," 6th CEAS Aerospace Europe Conference, CEAS 2017 Paper No. 2404.

[21] Schlösser, P., Meyer, M., Schüller, M., Weigel, P., and Bauer, M., "Fluidic actuators for separation control at the engine/wing junction," Aircraft Engineering and Aerospace Technology, Vol. 89, No. 5, 2017, pp. 709-718.

[22] Nagib, H., Kiedaisch, J., Reinhard, P., , and Demanett, B., “Active Flow Control for High Lift Airfoils: Separation Versus Circulation Control," 45th AIAA Aerospace Sciences Meeting and Exhibit, AIAA Paper 2007-1119, 2007. 